\title{
Estudio genético en gemelos
}

\author{
Vera Daher N. ${ }^{1}$; Ronald Youlton R. ${ }^{1}$; Julio Nazer $\mathrm{H}^{2}{ }^{2}$ Lucia Cifuentes $\mathrm{O}^{3}$
}

\section{Genetic studies in twins}

\begin{abstract}
Durjng the period june 1987 - january 1988, 35 consecutive pairs of twins born at two maternity hospltals in the northern area of Sentiago were studied to search evidence of genetic or other factors which could eventually favour twinning. Sixteen of these pairs ware considered to be monozygotic $\{M Z\}$ after studying five biood group systems (ABO, Rh, MNSs, Duffy and Kidd) applying the method of Smith and Penrose. Maternal age was higher in dizygotic twins (DZ) than in the $M Z$ group (average $30.1 \mathrm{yrs}$. $\mathrm{v} / \mathrm{s} 26.8 \mathrm{yrs}$, respextively (p $<0.05$ ). Family history of twinning was positive in $73 \%$ of the $M Z$ and in $82 \%$ of the $D Z$ twins. The Duffy and Kidd systems were not in Hardv-Weinberg equilibrium due to a deficit of heterozigotes in MZ as well as in DZ twins. There was a significant increase in MS and Ms haplotypes in both groups of twins respect to the chilean general population ip = 0.01). These results suggest the presence of a genetic determinant that favors the production of twinning of DZ as well as MZ type. A larger number of cases will be required to test this hypothesis.

(Key words: twins, dizigotic, monozigotic, maternal age, family history, blood groups, genetic studies.)
\end{abstract}

F. Galton, en 1876, fue el primero en recopilar observaciones en gemelos y obtener información sobre la participación de la herencia y del ambiente en las diferencias individuales ${ }^{1}$.

El estudio del posible origen zigótico de los gemelos, que denominaron zigocidad, en una población determinada, se realiza con la finalidad de tener mejor conocimiento en ella de las frecuencias de gemelaridad, mascadores genéticos, polimorfismos $y$ de la relación que pudiese existir entre la incidencia de gemelarjdad con ciertos factores genéticos y no genéti$\cos ^{2-4}$.

Los gemelos se estudian y se clasifican basándose en el sexo, marcadores genéticos y tipos de placenta. En forma genérica se les clasifica como idénticos o monozigóticos (MZ) y fraternos o dizigóticos (DZ). En el caso de los $M Z$, la frecuencia es aproximadamente de 1/250 nacimientos; ellos son producidos por la fertili-

1. Servicio de Genética, Departamento de Medicina. Hospital Clínico José Joaquín Aguiree, Univetsialad de Chile.

2. Unidad de Neonatología, Departamento de Obstetricja y Ginecología. Hospital Clínico José Joaquin Aguirre. Universidad de Chile.

3. Departamento de Biología Celular y Genética. Facultad de Medicina. Universidad de Chile. zación de un solo óvulo por un espermio, pero donde el huevo resultante se divide posteriormente en dos, que desarrollan cada uno un feto con idéntica composición genética. Los melljzos fratemos (DZ) son dos veces más comunes que los jdénticos y son productos de doble ovulación, caso en que cada óvulo es fertilizado por distintos espermios, formándose dos fetos que genéticamente son como dos tipicos herma$\operatorname{nos}^{3,5-7}$. Otras formas de gemelaridad han sido descritas, pero son de rara ocurrencia ${ }^{3}$.

E] origen monozigoto no puede ser establecido con certeza absoluta en gemelos idénticos (MZ), pero se puede calcular su probabilidad utilizando el método de Smith y Penrose u otros similares ${ }^{8-10}$.

Diversos factores influyen en la incidencia de gemelaridad, algunos de los cuales son bien conocidos para los DZ, pero son un enigma para los $\mathrm{MZ}^{2-5}$.

La funalidad de este trabajo fue determinar las proporciones de $\mathrm{MZ}$ y $\mathrm{DZ}$ entre gemelos nacidos consecutivamente en matemidades de Santiago Norte, analizar ciertos posibles factores determinantes, discutir la relación de éstos con la incidencia de gemelaridad y comparar las frecuencias génicas de diversos grupos sanguineos de los gemelos con las de una experiencia previa del area Norte de Santiago ${ }^{11}$. 


\section{Material y Método}

En el perf́odo entre junio de 1987 y enero de 1988 , en el Servicio de Genética del Hospitaj Clinico José Joaquín Aguirre, de la Universidad de Chile, se tealizó un estudio prospectivo de zigocidad en gemelos, para lo cual se consideraron, en forma consecutiva, 35 pares de gemelos nacidos en exe período en las maternidades del mismo hospital (19 pares) y del hospital San José, del Ministeric de Salud (16 pares).

Se confeccionó una ficha especial que contenía información del recién nacido; historia obstétrica de la madre (número de hijos, edad y sexo de cada uno), método anticonceptivo empleado previamente; identificación y nivel socjoeconómico de los padres (edad, ocupación, raza, antecedentes de consanguinidad); antecedentes de gemelos en la familia (para lo cual se confécionó la genealogía).

4 partir de una muestra de sangre obtenida del cosdón umbilical sin anticoagulantes, se realizó tipificación en los sigujentes grupos sanguíneos: ABO, $\mathrm{Kh}$ (CDEce), MNSs, Duffy a, Duffy b, Kidd (jka, jkb). Se utilizó, para cada determinación, un panel de glóbulos rojos estándar para controlas la calidad de los antisueros.

Se estimó la zigocidad de los gemelos considerando el sexo y los diversus grupos sanguíneos y se les clasificó como DZ y MZ. Se utilizó el método de Smith y Penrose para calcular la probabilidad de ser $\mathrm{MZ}^{\mathrm{s}}$ y prueba de $Z$ para determinar la significación estadística $D Z$ vs. $M Z$ para los antecedentes de gemelos en la familia y el método anticonceptivo empleado ne viamente por las madres. La intluencia de las edades materna y paterna para la ocurrencia de $\mathrm{DZ}$ vs. MZ se evaluó mediante prueba de $t$. Las frecuencias génicas de los sistemas $A B O$, Rh y MNSs se estimaron a través del método de máxima verosimilitud. En el caso de los sistemas Duffy y Kidd la estimación se hizo directamente a partír de la muestra. Se evaluó la existencia de equilibrio Hardy Weinberg en cada sistema sangufneo mediante prueba de ji cuadrado $\left(x^{2}\right)$.

Se comparazon las frecuencias génicas de los distintos grupos sanguíneos de los gemelos $\mathbf{M Z}$ y $\mathbf{D Z}$ con las descritas por una experíencia previa en población general de la misma área de salud ${ }^{\mathrm{t}}$.

\section{Resultados}

De los 35 pares de gemelos estudiados, 25 tenian igual sexo y de éstos, 16 iguales grupos sanguíneos; a este últíno grupo se le calculó la probabilidad de ser $M Z$, resultando en todos mayor o igual a $99 \%$, por lo que fueron clasificados como gemelos idénticos de origen monozigotos. Los restantes 9 pares del mismo sexo fueron distintos en los grupos sanguineos. Otros diez pares tenían distinto sexo; de éstos, 4 eran iguales en los grupos sanguineos y 6 distintos. Por lo tanto, 19 pares de gemelos resultaron ser distintos en sexo o grupos sanguíneos o ambos, clasificándolos como dizigotos de origen.

De los 16 pares de gemelos $\mathbf{M Z}, 8$ tenían sexo femenino (F) y 8 masculino (M). Entre los 19 pares DZ, 10 eran de distinto sexo y 9 del mismo $(4 \mathrm{~F}, 5 \mathrm{M})$.

El conjunto de la muestra de gemelos fue homogéneo desde el punto de vista de condición socioeconómica y étnica.

El antecedente de uso de anticunceptivos hormonales antes del embarazo múltiple estaba presente en $56,2 \%$ de $\operatorname{los} \mathrm{MZ}$ y en $50 \%$ de los DZ, la que no inostró significación estadística (p $>0,05)$.

La media de la edad de los padres en Jos $\mathrm{MZ}$ fue de 31,5 años y los DZ 30 ar̃os, lo que tampoce tiente significación estadistica. Sin embargo, la media de la edad de las madres en los MZ fue de 26,8 años $y$ en los DZ 30,1 años, diferencia para la cual la prueba de $t$ dio 1,675 (33 gl), significativo al $5 \%(\mathrm{p}<0,05)$ (figura).

Al comparar las curyas $M Z$ y $D Z$ en la figura, los DZ muestran una cúspide de frecuencia en el rango de edad de las madres entre 30 y 40 años, mientras en los $\mathrm{MZ}$ el nuayor número $(68 \%)$ se da en el rango de 25 a 29 años de edad matema.

El antecedente da gemelos en la familia se re. gistró en $73 \%$ Je los pares $M Z$ y $82 \%$ de los DZ. En ambos tipos de gemelos predominá el antecedente por linea materna: $9 / 16(56 \%)$ en los MZ y $13 / 19(68 \%)$ en los DZ.

En la tabla 1 se aprecian las frecuencias gé. nicas de los grupos sanguineos estudiados en las muestras de gemelos MZ y DZ y de la pobla. ción del área norte de Santiago previamente descrita. Ellas fueron similares en los tres conjuntos de sujetos para los sistemas $\mathrm{ABO}, \mathrm{Rh}$, Duffy y Kidd. Sin embargo, las frecuencias haplotipicas para el sistema MNSs, fueron estadisticamente diferentes $\left(\chi^{2}=21,69, p=0,01\right)$ y obedecen a mayor frecuencia de MS y $\mathrm{Ms}$ en los gemelos, especialmente los DZ.

Los dos grupos de gemelos $\mathrm{MZ}$ y $\mathrm{DZ}$ están en equilibrio de Hardy.Weinberg para los sistemas ABO, Rh y MNSs. En el caso del sistema Duffy, tanto los gemelos $\mathrm{MZ}$ y $\mathrm{DZ}$ se alejan significativamente del equilibrio, debido a es. casez de heterozigotos (tabla 2). Para el sistema Kidd ocurre algo similar: los gemelos se akjan del equilibrio de Hardy-Weinberg debido a gran deficiencia de hetcrozigotos, especialmente los DZ en que la carencia de éstos es total (tabla 3). 


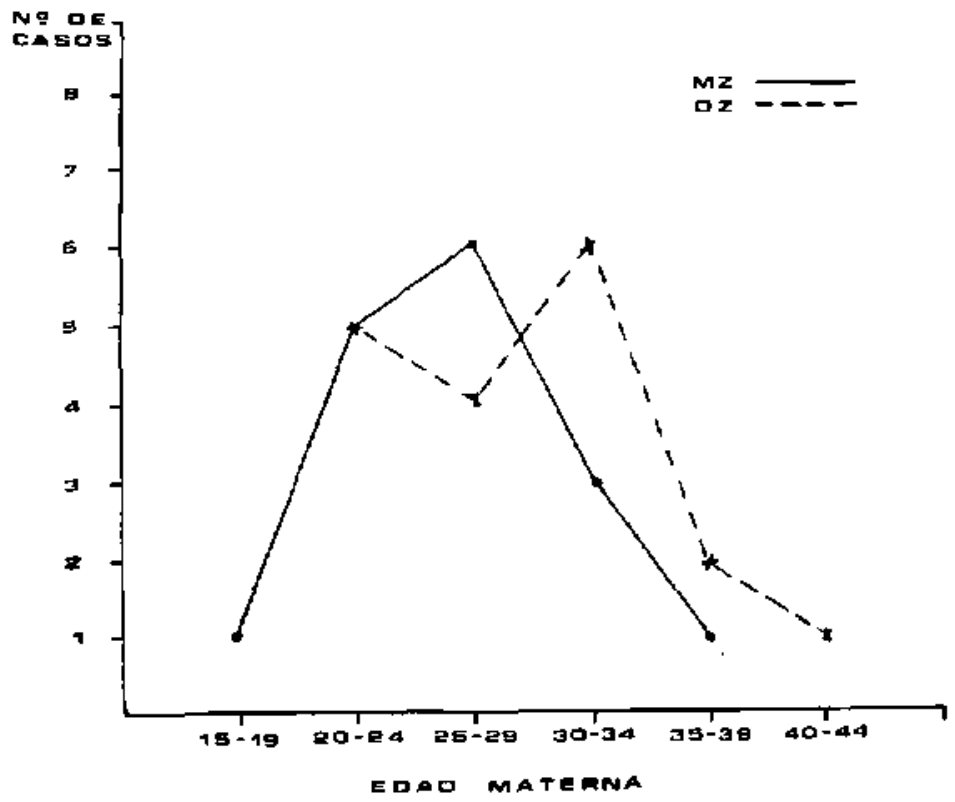

Figura: Distribución de la edad materna en 16 pares de gemelos monozigotos y 19 pares de gemelos dizigotos.

Tabla 1

Estudio de gemelos (frecuencias génicas)

\begin{tabular}{|c|c|c|c|c|}
\hline & & Monuzigotos & Dizigotos & A. Norte \\
\hline \multicolumn{5}{|l|}{ Sistema ABO } \\
\hline A & & 0,2069 & 0,1895 & 0,1724 \\
\hline B & & 0,0638 & 0,0268 & 0,0580 \\
\hline \multirow[t]{2}{*}{0} & & 0,7293 & 0,7837 & 0,7696 \\
\hline & n: & 32 & 38 & 65.309 \\
\hline \multicolumn{5}{|l|}{ Sistema Rh } \\
\hline CDe & & 0,4063 & 0,5263 & 0,5001 \\
\hline CDE & & 0,2813 & 0,2368 & 0,2259 \\
\hline \multirow[t]{2}{*}{ cde } & & 0,3125 & 0,2368 & 0,2192 \\
\hline & $\mathrm{n}:$ & 32 & 38 & 1.429 \\
\hline \multicolumn{5}{|l|}{ Sistema MNSs } \\
\hline MS & & $0,1752^{*}$ & $0,2771^{*}$ & $0,1378^{*}$ \\
\hline Ms & & 0,3873 & $0,4993^{*}$ & 0,3873 \\
\hline NS & & 0,0435 & 0,0256 & 0,0927 \\
\hline \multirow[t]{2}{*}{$\mathrm{N}_{\mathrm{s}}$} & & 0,3939 & 0,1981 & 0,3820 \\
\hline & $\mathbf{n}:$ & 32 & 38 & 258 \\
\hline \multicolumn{5}{|l|}{ Sistema Duffy } \\
\hline FY (a) & & 0,5938 & 0,6053 & 0,5213 \\
\hline \multirow[t]{2}{*}{$\Gamma Y^{\prime}(b)$} & & 0,4062 & 0,3947 & 0,4787 \\
\hline & $n:$ & 32 & 38 & 684 \\
\hline \multicolumn{5}{|l|}{ Sistema Kidd } \\
\hline JK (a) & & 0,4583 & 0,5333 & 0,4615 \\
\hline \multirow[t]{2}{*}{$J K(b)$} & & 0,5417 & 0,4667 & 0,5385 \\
\hline & $\mathbf{n}:$ & 24 & 30 & 182 \\
\hline
\end{tabular}

* Prueba de $X^{2}$ DZ vs. área Norte

MZ vs. área Norte significativo $p=0,01$

DZ vs. MZ

$\mathbf{n}=$ total individuos. 
Tabla 2

Frecuencias fenotípicas del sistema Duffy en gernelos monozigotos $y$ dizigotos

\begin{tabular}{|c|c|c|c|c|c|c|}
\hline \multirow[t]{2}{*}{ Fenotipo } & \multicolumn{3}{|c|}{ Monozigotos } & \multicolumn{3}{|c|}{ Dizigotos } \\
\hline & Obs. & Esp. & $x^{2}$ & Obs. & Esp. & $x^{2}$ \\
\hline Fy $(a+b-)$ & 14 & 11,3 & 0,65 & 18 & 13,9 & 1,19 \\
\hline Fy $(a+b+)$ & 10 & 15,4 & $1,91^{*}$ & 10 & 18,16 & 3,67 \\
\hline \multirow[t]{3}{*}{ FY $(a-b+)$} & 8 & 5,3 & 1,39 & 10 & 5,92 & 2,80 \\
\hline & \multicolumn{3}{|c|}{$x_{1}^{2}=3,97$} & & \multicolumn{2}{|c|}{$x^{2}=7.67$} \\
\hline & \multicolumn{3}{|c|}{$p=0,046$} & & \multicolumn{2}{|c|}{$\mathrm{p}=0,001$} \\
\hline
\end{tabular}

* Indica déficit de heterozigotos.

Obs. = observado.

Esp. $=$ esperado.

Tabla 3

Frecuencias fienot ípicas del sistema Kidd en genelos monozigotos y dizigotos

\begin{tabular}{|c|c|c|c|c|c|c|}
\hline \multirow[t]{2}{*}{ Fenotipo } & \multicolumn{3}{|c|}{ Monozigotos } & \multicolumn{3}{|c|}{ Dizigotos } \\
\hline & Obs. & $E_{5 p}$ & $x^{2}$ & Obs. & Esp. & $x^{2}$ \\
\hline $\mathrm{jK}(\mathrm{a}+\mathrm{b}-)$ & 8 & $s$ & 1,74 & 16 & 8,5 & 6.5 \\
\hline jK $(a+b+)$ & 6 & 11.9 & $2,94 *$ & 0 & 14,9 & $14.9 *$ \\
\hline \multirow[t]{3}{*}{$\mathrm{j} K(\mathrm{a}-\mathrm{b}+)$} & 10 & 7 & 1,24 & 14 & 6,5 & 8,5 \\
\hline & \multicolumn{3}{|c|}{$\begin{array}{l}x^{2}=5,91 \\
1\end{array}$} & & \multicolumn{2}{|r|}{$\begin{array}{l}\chi^{1}=30 \\
1\end{array}$} \\
\hline & \multicolumn{3}{|c|}{$p=0,015$} & & \multicolumn{2}{|r|}{$p=\begin{array}{r}(-7) \\
<10\end{array}$} \\
\hline
\end{tabular}

Obs, = observado.

Esp. $=$ esperado.

* = déficit óe heterozigotos.

\section{Discusión}

Los factores más conocidos que pueder predisponer a la ocurrencia de gemelos DZ son: la edad materna, el grupo étnico, el antecedente de gemelos en la familia y factores tales como uso de anticonceptivos hormonales, fertilización in vitro y métodos de inducción de ovulación ${ }^{2-5}$. En el caso de los gemelos $\mathrm{MZ}$ los factores condi. cionantes aún no están claros y no hay eviden. cias de aspectos genéticos que los determinen 3,56 .

$\mathrm{Si}$ bien la mayoría de los $\mathrm{MZ}$ son genéticamente idénticos y tienen el mismo cariotipo, se han descrito casos en que éste es diferen$\mathrm{te}^{3,8,12,13}$, como, por ejemplo, un gemelo con cariotipo normal y el otro con monosomia $X(45, X)$; u otro con un cromosoma $X$ adicional (XXY), o bien una anomalía estructural del cromosorna 18 (del 18, ) hechos que son explicables por no disyunción o delección cromosómica postzigótica $^{3,12}$.

Los agentes inductores de ovulación aumen. $\tan$ la incidencia de gemelos DZ. Se ha descrito que tambièn aumentarían la frecuencia de los $\mathrm{MZ}^{4}$; la explicación para este último fenómeno continúa siendo especulativa. Hasta el momento los otros factores que influyen en el aumento de incidencia de los $D Z$, no estarían relacionados con la producción de MZ, por lo que ambos grupos son considerados distintos, en cuanto al mecanismo por el que ocurren. En este estudio 
hemos analizado algunos factores usando una muestra de gemelos DZ y $\mathrm{MZ}$ homogénea en cuanto a grupo étnico y nivel socioeconómico.

El método de Smith y Penrose empleado para la determinación de la zigocidad ${ }^{8}$ con los marcadores genéticos utilizados, da grados de certeza superiores a $99 \%$ para la monozigocidad, lo que consideramos suficiente para nuestro propósito.

El hecho de que no hubiese diferencias significativas en el uso de anticonceptivos hormonales entre las madres de gemelos $\mathrm{MZ}$ y $\mathrm{DZ}$ podría ser explicable por la alta frecuencia con que éstos son utilizados por la población.

La mayor edad materna observada en los ge. melos DZ coincide con lo descrito en diversos grupos étnicos ${ }^{2}, 3$.

La relación de los DZ con la edad materna fue reconocida por Weinberg en $1901^{2}$ y podria ser explicada por el aumento de la hormona folículo estimulante (FSH) observada a edades avanzadas?. La sobreestimulación hormonal seria la responsable de la ovulación múltiple, tal cual ocurre en los tratamientos de inducción de ovulación ${ }^{4 \div 6}$.

La incidencia máxima de gemelos $\mathrm{MZ}$ en el grupo etario 20 a 29 años guarda, en cambio, estrecha relación con la proporción de partos a la misma edad en nuestro pais ${ }^{14}$.

La concentración de nacimientos de gemelos en ciertas familias también fue reconocida por Weinberg ${ }^{2}$, asimilándola a un modelo de herencia multifactorial. El antecedente de gemelos en la familia en nuestra serie se presentó con una frecuencia mayor de $70 \%$ en ambos grupos, predominando el antecedente por línea materna. Una alta frecuencia repetitiva de gemelos en una familia, no implica que la gemelaridad sea un rasgo heredable, más bien sugiere que las madres de gemelos tendrían una constitución genética que las haría más susceptibles a responder frente a ciertos agentes externos que les provocasen. por ejemplo, ovulaciones múltiples $^{2,3,6}$, E1 anteçedente de gemelos a través de la línea materna, observado en nuestros casos $M Z$, no lo hemos encontrado descrito en la lite. ratura revisada.

En los sistemas sanguíneos de $\mathrm{MZ}, \mathrm{DZ}$ y la población general del área norte de Santiago, se encontraron mayores frecuencias génicas de los haplotipos MS y Ms en los gemelos, especialmente en los DZ.

El alejamiento para los sistemas Duffy y Kidd, del equilibrio de Hardy-Weinberg, observado en ambos tipos de gemelos, se explica por deficiencia de heterozigotos, y tampoco lo hemos encontrado descrito en la literatura.

Nuestros resultados inducirían a pensar en la existencia de un mecanismo genético de control - producción de gemelaridad que actuaria tanto para los $\mathrm{MZ}$ como para los DZ. Sin embargo, estos resultados, por lo reducido de la muestra, no son concluyentes. Pretendemos continuar con este estudio ampliando nuestra muestra para confirmar o rechazar esta hipótesis.

\section{Resumen}

Durante el período junio 1987 a enero 1988 es estudiaron. en forma consecutiva 35 pares de gemelos nacidos en dos maternidades de hospitales del área Norte de Santiago, con el propó. sito de buscar evidencia de factores genéticos que eventualmente influyen en las posibilidades de gemelaridad, comparando las frecuencias génicas de diversos grupos sanguíneos entre gemelos monozigóticos, mellizos dizigóticos y controles de una misma área geográfica. Dieciséis de ellos fueron considerados monozigóticos (MZ) después del estudio de cinco sistemas de grupos sanguíneos ( $\mathrm{ABO}, \mathrm{Rh},(\mathrm{C}, \mathrm{D}, \mathrm{E}, \mathrm{c}, \mathrm{e})$, MNSs, Kidd. Duffy), aplicando el método de Smith y Penrose. La edad materna fue más alta en los mellizos dizigóticos (DZ), que en los monozigóticos $(\bar{x}=30,1$ años vs. 26,8 años. respectivamente). La historia familiar de gemelaridad fue positiva en $73 \%$ de MZ y $82 \%$ de DZ. Los sistemas Duffy y Kidd no estaban en equilibrio de Hardy.Weinberg, debido a déficit de heterozigotos para dichos caracteres entre los MZ y también los DZ. Se encontraron significativamente aumentados los haplotipos MS y Ms en ambos grupos de mellizos con respecto a una población general de la misma área geográfíca. Estos resultados sugieren la presencia de un determinante genético que favorece la producción de gemelaridad de DZ como también de MZ. Se requeriría un mayor número de casos para probar esta hipótesis.

(Palabras clave: gemelos, dizigotos, monozigotos, grupos sanguíneos).

\section{Referencias}

1. Becker, P.E.: Genética humana. Barceiona: Edicjones Toray S.A. 1968;10-85. 
2. Serrn Curt.: Principles of human genetics. Second Edition. San Francisco and London: Freeam and Company $1960 ; 530-573$.

3. Thompson, I.: Thompson, M.: Genetics in Medicine. Fourth Edition, Philadelphia: W.B. Saunders Company 1986;273-280.

4. Deron, ' $:$ Deron, R.; Vlietinck, R.; Van Derberche. $H$. : Thiery. $M$ : Increased monozygotic twinning rate after ovtilation induction. Lancet 1987 ; I: $1236-1238$.

5. Ghat, V.: Volyosagard. D.: Morbility and mortality factors in twins. Twin pregnancy. Clin Perinatol $1988 ; 15: 123-140$.

6. Voyel, F.: Motulsky. A.: Human Genetics. Second Edition Heidelberg: Springer Verlag 1986; 20S$2] 2$.

7. Atkinsom, Di Ftecuency dizigotic twinning. Nature $1986 ; 322: 780$

8. Smith, S.M.: Penrose, L.S.: Monozygotic and dizygotic. Twin diagnosis. Ann Hum Genet 1955 19:273-289.

9. Valenzuela, C.: Programa general para calcular la probabilidad de dizigosis en sistemas genéticos multialélicos. Rev Med Chile 1980; 108: 1143. 1146.

10. Cruz-Coke, R.; Armanet, L., Lyns, $C_{\text {. }}$ Valenzucla, $C$.: Tabla chilena para el diagnústico de zigocidad en gemelos. Rev Med Chile 1980; 108605 .

11. Cifumes, L.: Valenzuela, $C .:$ Cruz-Coke, $R .:$ Armonet, l.: l.'mg, C.; Harb, Z.: Caracterización genética de la población hospitalaria de Santiago. Rev Med Chile 1988; 116: 28-33.

12. Flannery', $D$; Holsmon, $G_{*}$ : Prenatal resting and twinning, Clin Genet 1985;28: 180-182.

13. Uchida, f.; Freenan, $V_{\text {.: }}$ Triploidy and chromosonnes. Am J. Obstet Gynecol 1985; 1: 65-69.

14. Anónimo. Institutu Nacional de Estadística, Santiago, Chile, 1983. 